\title{
Optimization of in vitro seed germination of Taraxacum platycarpum
}

\author{
Jung-Hwan Lee ${ }^{1 \dagger \dagger}$, Young-Kwan Kim ${ }^{1) \dagger}$, Eun-Yi $\mathrm{Oh}^{1)}$, Kuk-Young Jung ${ }^{2)}$, and Kisung $\mathrm{Ko}^{1)^{*}}$ \\ ${ }^{1)}$ Department of Biological Science, College of Natural Sciences, Wonkwang University, Iksan 570-749, Korea \\ ${ }^{2)}$ Jungkukyoung Oriental Clinic, Suwon 441-872, Korea \\ (Received December 11, 2009, Accepted December 25, 2009)
}

\begin{abstract}
Dandelion (Taraxacum platycarpum) has been widely utilized for medicinal purposes. However, the dandelion seeds are relatively difficult to germinate under cultivation conditions, which hampers seedling propagation of dandelion plants and reduces the opportunity of usage of such a useful medicinal plant. Thus, in this study, in vitro conditions for the dandelion seed gemination were optimized to enhance the germination rate. In seed washing steps, the sequential treatments with $20 \%$ of ethanol, $20 \% \mathrm{of} \mathrm{NaOCl}$, and distilled water avoided microbial contamination with the highest in vitro germination rate $(67.5 \%)$ from seeds sown in germination media. The media supplemented with $1.4 \mathrm{~g} / \mathrm{L}$ of MS salts and $1 \%$ of sucrose significantly enhanced the germination rate compared to the media with $4.4 \mathrm{~g} / \mathrm{L}$ of $\mathrm{MS}$ and $3 \%$ of sucrose. Sowing the seeds vertically in the optimized media supplement conditions, $1.4 \mathrm{~g} / \mathrm{L}$ of MS salts and $1 \%$ of sucrose, gave the maximum in vitro germination rate $(61 \%)$, which was almost three times higher than sowing seeds on a soil pot $(23 \%)$. Our results indicate that the seed washing and sowing methods including germination medium supplements can be optimized to enhance in vitro seed germination of dandelion.
\end{abstract}

Key Words: Dandelion, Germination, Seedling, Surface sterilization, Taraxacum

\section{INTRODUCTION}

Dandelion plants, the genus Taraxacum, a member of the family Asteraceae is the perennial plant with a stout taproot and rosette-type leaves, which is world widely found in the warmer temperate zones. In Korea, many varieties including $T$. coreanum, $T$. hallaisanensis, T. officianale, T. ohwianum, and T. platycarpum are broadly distributed. T. platycarpum has been utilized in traditional Korean herbal medicine for pharmacological properties including their diuretic, choleretic, anti-inflammatory, and anti-carcinogenic activities ${ }^{1)}$. Recent studies have shown that it is high in vitamin $C$ and potassium compared to other leaf vegetables ${ }^{2}$. In addition, its extract has ant-microbial and anti-coagulant activities, ${ }^{3,4}$, and contains desacetylmatricarin, an anti-allergic component ${ }^{5)}$. Thus,

\section{*연락저자:}

Tel: +82-63-850-6088; Fax: +82-63-857-8837

E-mail: ksko@wku.ac.kr

${ }^{\dagger}$ These authors contributed equally to this paper. consumption of medicinal dandelion plants is prevalent and growing in Korea. However, propagation and cultivation of most herbal plants including dandelion are difficult because of low germination rates or specific environmental requirements ${ }^{6}$. Although, dandelion seeds can germinate without long-period of dormancy, it is difficult to optimize environmental conditions for seed germination. Sometimes, chilling treatment at a certain temperature required for seed germination is considered as an essential to success ${ }^{7}$. Low germination rates of dandelion seeds frequently are due to pathogenic infection or physical and physiological damage to seeds ${ }^{8)}$. In addition, its seedling establishment rate is very low $(1 \%)^{9)}$. Thus, dandelion is often vegetatively propagated using leaf cutting methods ${ }^{10)}$. The controlled growth conditions under the hydroponic systems or in vitro plant tissue or cell suspension culture systems could allow us to control the contents of important medicinal compounds and their potency ${ }^{8}$. Furthermore, in vitro culture systems are feasible to be manipulated to 
increase potency of active compounds through optimized culture conditions and enhance standardization in dandelion plant production. Even such systems can avoid unfavorable compounds including heavy metal soil contaminants, which represent an emerging risk to public health ${ }^{11)}$. In this study, we investigated in vitro seed germination under different seed washing methods as a pre-treatment for seedling tissue culture and the different media supplements and sowing methods to optimize in vitro conditions for increasing the germination rate and establishing seedling of dandelion (T. platycarpum).

\section{MATERIAL AND METHODS}

\section{Plant seed materials}

Seed washing preparation, sowing method, and in vitro germination culture were conducted using some modifications of the protocols previously reported ${ }^{12-14}$. Dandelion seeds (Taraxacum platycarpum H. Dablstaed) were washed, and only viable seeds, determined by floatation method, were used. The seeds were air-dried for a couple of hours and only properly filled seeds were sorted out for use. Four hundred well-filled seeds were finally selected for the experiments.

\section{Seed washing methods}

To investigate the effect of concentration (20 and $70 \%$ ) of ethanol and (1 and $20 \%$ ) of sodium hypochlorite $(\mathrm{NaOCl})$ in surface sterilization washing buffer on seed germination, seeds were washed with only sterile distilled water (control), 20\% of ethanol and distilled water $(20 \% \mathrm{E}), 70 \%$ of ethanol and $1 \%$ of $\mathrm{NaOCl}$, and distilled water $(70 \% \mathrm{E}+1 \% \mathrm{~N})$, and $20 \%$ of ethanol, $20 \%$ of $\mathrm{NaOCl}$, and sterile distilled water $(20 \% \mathrm{E}+20 \% \mathrm{~N})$, respectively. All sequential washing steps were conducted for $2 \mathrm{~min}$. There were, therefore, 4 treatment combinations, each replicated three times, and each replicate was sown with twenty seeds. Four hundred seeds were sequentially soaked and washed for $2 \mathrm{~min}$ in each sterilization solution.

\section{Seed germination media and seed sowing methods}

After washing treatment, the seeds were moved onto a Whatman filter paper (No.1) and dried at room temperature. Thereafter, seeds were germinated in media supplemented with Murashige and Skoog
(MS) salts and sucrose in long day conditions consisting of $16 \mathrm{~h}$ of light with a light intensity of $20 \mu \mathrm{mol} / \mathrm{m} / \mathrm{s}$ from $40 \mathrm{~W}$ cool white and red deluxe fluorescent tubes (1:1 mix) and $8 \mathrm{~h}$ of darkness at $26^{\circ} \mathrm{C}$, respectively. To optimize germination medium conditions, the surface-sterilized seeds were germinated on two different media supplemented with MS salts $(1.4$ or $4.4 \mathrm{~g} / \mathrm{L}$ ) and sucrose $[1$ or $3 \%(\mathrm{wt} / \mathrm{vol})]$. In addition, to compare the effects of seed sowing position, seeds were sown horizontally or vertically on the media. The germination seed rates were observed on day 10, 13, 16, 19, 22, and 25 to investigate the effect of the seed sowing position and medium conditions varied with concentration of MS and sucrose on the seed germination rate of dandelion.

\section{Statistical Analysis}

The seed germination rate and contamination rate were expressed as a percentage, which was calculated by using the following equation: (number of germinated seeds or contaminated seeds/total number of seeds per Petri dish) X 100. The data represent the means for three replicate samples. Error bars indicate standard deviations (SD). Student's t-tests were performed to determine the statistical difference in the germination rates between two treatments using the Instat 2.03 statistical package (GraphPad Software, San Diego, CA).

\section{RESULTS}

\section{Optimization of seed washing buffer to control contamination}

Dandelion seeds were washed in different solutions before seedling on the tissue culture medium and their contamination rates were investigated (Fig. 1). The contamination rate of seeds washed with only distilled water was $100 \%$. In seeds washed with $20 \%$ of ethanol, the contamination rate $(34 \%)$ was dramatically decreased. The contamination rate $(25 \%)$ was further decreased in seeds washed with $70 \%$ of ethanol and $1 \%$ of $\mathrm{NaOCl}$. However, it seemed to be directly no contamination from seeds washed sequentially with $20 \%$ of ethanol, $20 \%$ of $\mathrm{NaOCl}$, and distilled water.

\section{Effect of washing buffer on germination rate}

The in vitro germination rate was observed in seeds treated with different washing protocols (Fig. 1). 


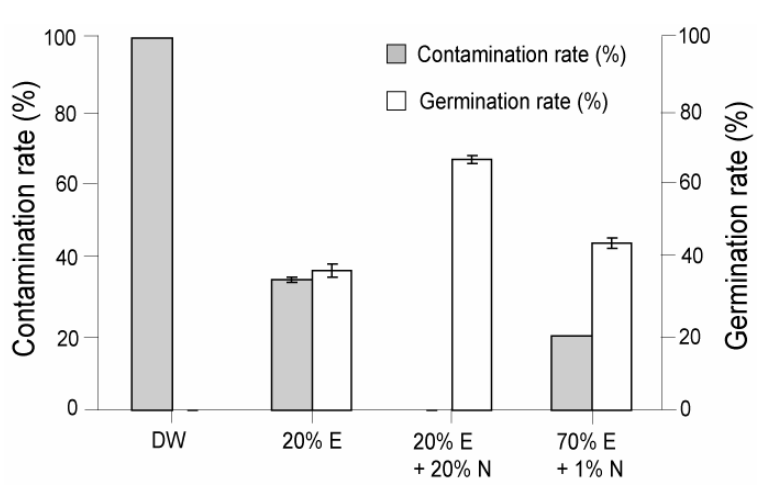

Fig. 1. Effect of seed washing buffer on in vitro contamination and germination rates. DW, seeds washed with distilled water; $20 \% \mathrm{E}$, seeds washed sequentially with $20 \%$ of ethanol and DW; $20 \% \mathrm{E}+20 \% \mathrm{~N}$, seeds washed sequentially with $20 \%$ of ethanol, $20 \%$ of $\mathrm{NaOCl}$, and DW; $70 \%$ $\mathrm{E}+1 \% \mathrm{~N}$, seeds washed sequentially with $70 \%$ of ethanol, $1 \%$ of $\mathrm{NaOCl}$, and DW.

No seed with distilled water treatment was germinated. In seeds washed sequentially with $20 \%$ of ethanol and distilled water, the germination rate was $40 \%$. The sequential treatment of $70 \%$ ethanol, $1 \% \mathrm{NaOCl}$, and distilled water slightly increased the seed germination rate $(45 \%)$. However, the highest germination rate $(67.5 \%)$ was observed in the seeds washed sequentially with $20 \%$ of ethanol, $20 \%$ of $\mathrm{NaOCl}$, and distilled water (Fig. 1).

Time courses of the cumulative seed germination on different in vitro media

The seed germination rate was observed at days after seed planting on two in vitro germination media [1.4 $\mathrm{g} / \mathrm{L}$ of MS salts and $1 \%$ of sucrose (' $\mathrm{A}$ ' media) and $4.4 \mathrm{~g} / \mathrm{L}$ of MS salts and $3 \%$ of sucrose ('B' media)] (Fig. 2). The germination rate of seeds planted on the ' $A$ ' media significantly higher compared to the ' $B$ ' media during all observed period after seed sowing. In seeds with the 'A' media, the cumulative germination rate rapidly increased from 10 days to 22 days after seed sowing. After 22 days, the germination rate was steady. In seeds with the ' $\mathrm{B}$ ' media, the germination rate also dramatically increased from 10 days to even 25 days after seed sowing. However, at 25 days, the cumulative seed germination rate with the ' $A$ ' media was significantly higher than the ' $B$ ' media (Fig. 2).

Effect of seed sowing position on germination rate

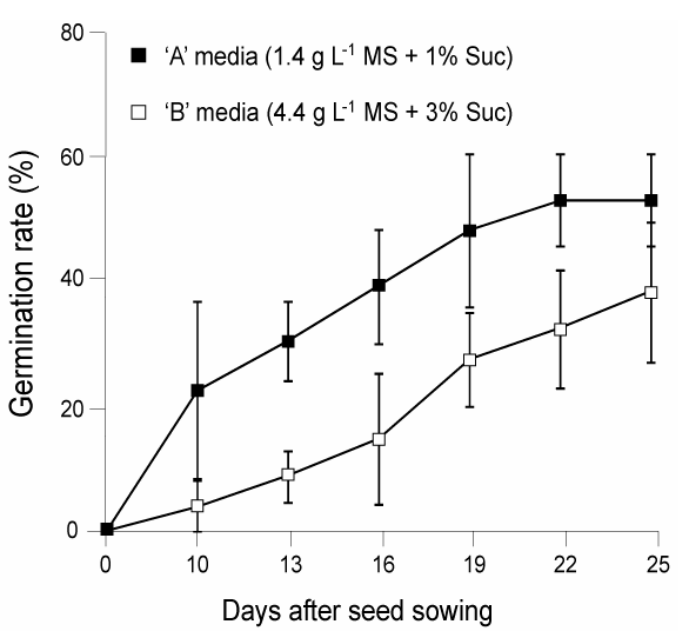

Fig. 2. Time courses of the cumulative germination rate of seed sown in two different in vitro media during 25 days after seed sowing. ' $A$ ' was seeds sown in media supplemented with $1.4 \mathrm{~g} / \mathrm{L}$ of MS salts and $1 \%$ of sucrose (Suc) (solid rectangle) whereas ' $B$ ' was seeds sown in media supplemented with $4.4 \mathrm{~g} / \mathrm{L}$ of MS salts and $3 \%$ of sucrose (Suc) (open rectangle).

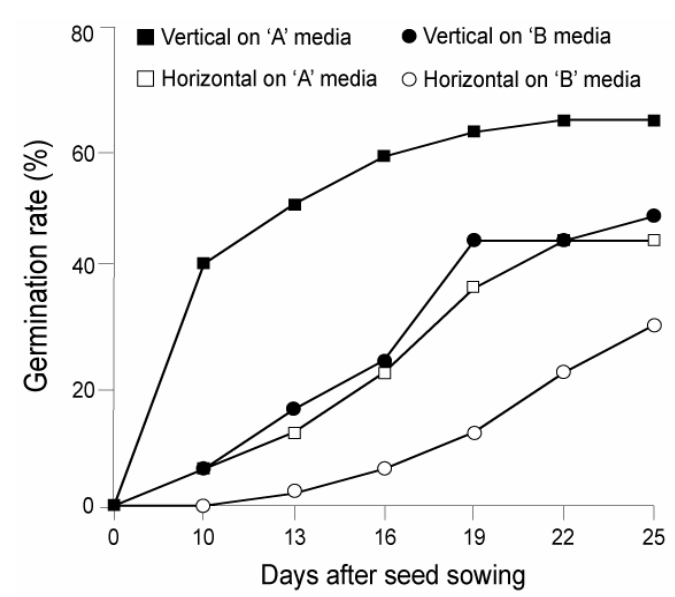

Fig. 3. Times courses of the cumulative germination rate of seed sown vertically and horizontally in media supplemented with different concentration of both MS salts (1.4 and $4.4 \mathrm{~g} / \mathrm{L}$ ) and sucrose (Suc) (1 and 3\%), respectively. The solid and open rectangles indicate the seeds sown vertically and horizontally on ' $A$ ' media supplemented with $1.4 \mathrm{~g} / \mathrm{L}$ of MS salts and $1 \%$ of Suc, respectively. The solid and open circles indicate the seeds sown vertically and horizontally on ' $B$ ' media supplemented with $4.4 \mathrm{~g} / \mathrm{L}$ of MS salts and $3 \%$ of Suc, respectively.

The final in vitro seed germination rates between horizontally and vertically sown seeds were statistically analyzed to investigate the effect of seed sowing position on germination (Fig. 3). In vertically and horizontally sown seeds on ' $A$ ' media, the mean 


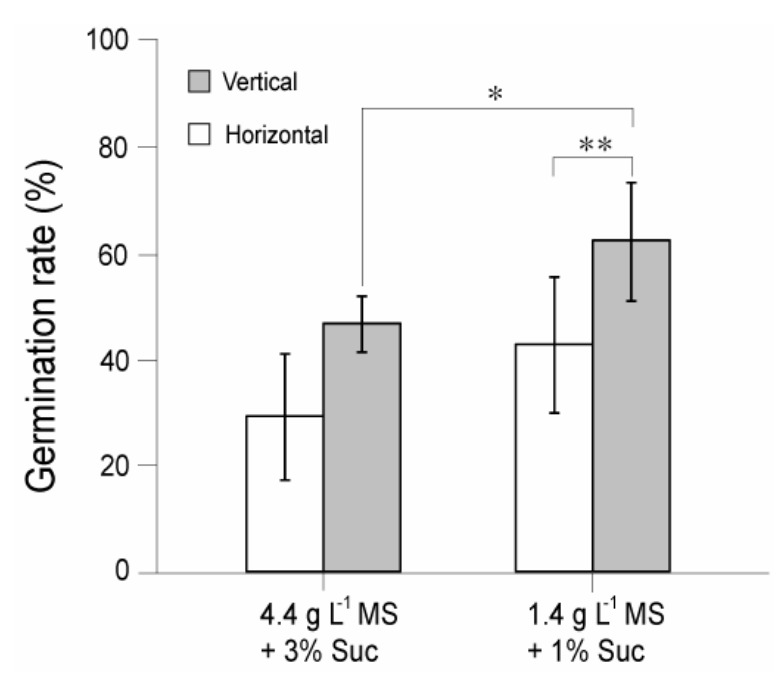

Fig. 4. Comparison of final germination rate of seed sown vertically and horizontally in different media conditions at 25 days after seed sowing. $1.4 \mathrm{~g} / \mathrm{L} \mathrm{MS}+\mathbf{1} \%$ Suc, 'A' media supplemented with $1.4 \mathrm{~g} / \mathrm{L}$ of MS salts and $1 \%$ of sucrose; $4.4 \mathrm{~g} / \mathrm{L} \mathrm{MS}+3 \%$ of Suc, 'B' media supplemented with $4.4 \mathrm{~g} / \mathrm{L}$ of MS salts and $3 \%$ of Suc. The symbols $(*$ and $* *)$ indicate statistical significant difference at $p<0.05$ and 0.01 , respectively.

values of the early germination rates at 10 days after planting seedling were $40 \%$ and $6 \%$, respectively. The mean value of the final in vitro seed germination rate of the vertical sowing position (64\%) was significantly higher compared to the rate of the horizontal seed sowing position (44\%) (Fig. 4). In vertically and horizontally sown seeds on ' $\mathrm{B}$ ' media, the mean values of the early germination rates at 10 days after planting seedling were $5 \%$ and $0 \%$, respectively. In ' $\mathrm{B}$ ' media, the mean value of the final in vitro seed germination rate of the vertical sowing position $(48 \%)$ increased compared to the rate of the horizontal sowing position (30\%). Regardless of the germination media, the vertical seed sowing position significantly enhanced the germination rate during the observed days after the seed sowing (Figs. 3 and 4).

\section{DISCUSSION}

Our present data demonstrate the effects of seed washing buffer, germination medium supplement concentration, and sowing position on the seed germination of dandelion (T. platycarpum). The main purpose of this study was to optimize those factors to enhance the in vitro seed germination rate of dandelion.

Since the seed germination study was conducted
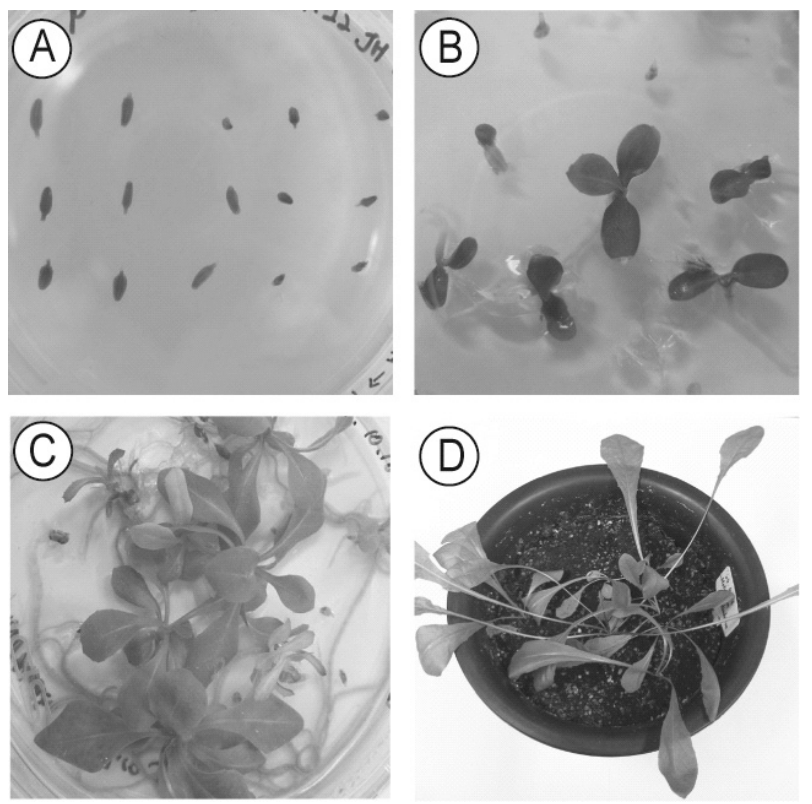

Fig. 5. Photoes of seed germination and seedling of Taraxacum platycarpum on in vitro medium and seeldings grown in a soil pot. Washed seeds were sown on medium (A). The in vitro germinated seeds (B) and in vitro seedlings $(C)$. The seedlings grown in a soil pot $(\mathrm{D})$.

under in vitro conditions, the seeds were washed before sown on the in vitro media plate (Fig. 5). The washing buffer affected the contamination rate of the dandelion seeds. Washing with only distilled water did not remove the contaminants from the seeds. Even the addition of only $20 \%$ of ethanol or $70 \%$ of ethanol, $1 \%$ of $\mathrm{NaOCl}$ and water did not avoid the microbial contamination from the seed germination media culture. The previous study where the dandelion seeds were washed sequentially with $70 \%$ of ethanol and $1 \%$ of $\mathrm{NaOCl}$, and distilled water did not report any contamination ${ }^{12)}$. However, in current study, such washing steps did not avoid the contamination. The sequential washing of seeds using $20 \%$ of ethanol, $20 \%$ of $\mathrm{NaOCl}$, and distilled water obtained complete removal of microbial contaminants. The seed germination rate was also affected by the washing buffer. Among four different washing buffers, the washing with only distilled water did not give any seed germination whereas the sequential washing with $20 \%$ of ethanol, $20 \%$ of $\mathrm{NaOCl}$, and distilled water showed the highest seed germination rate $(67.5 \%)$.

Time courses of the cumulative seed germination on two different in vitro media (' $\mathrm{A}$ ' and ' $\mathrm{B}$ ') regard of concentration of both MS and sucrose were compared 
to investigate the effect of both components on the seed germination. The ' $\mathrm{A}$ ' media supplemented with $1.4 \mathrm{~g} / \mathrm{L}$ of $\mathrm{MS}$ and $1 \%$ of sucrose significantly enhanced the seed germination rate compared to the ' $\mathrm{B}$ ' media containing $4.4 \mathrm{~g} / \mathrm{L}$ of $\mathrm{MS}$ and $3 \%$ of sucrose, indicating that the reduced concentration of both MS and sucrose in seed germination media increases the dandelion seed germination. These results are supported by a previous study where the media supplemented with 0 and $167 \mathrm{mM}$ of sucrose enhanced the Arabidopsis seed germination speed and showed the higher germination rate compared to the media with $333 \mathrm{mM}$ of sucrose ${ }^{15}$. Sugars play crucial roles in regulating seed germination through modulating both cellular abscisic acid (ABA) concentration and ABA response ${ }^{15)}$. Exogenously applied glucose increases the rate of $\mathrm{ABA}$ synthesis in seed, consequently reducing the seed germination ${ }^{15)}$.

Furthermore, the effect of seed sowing position on germination rate was investigated. Regardless of the concentration of both MS and sucrose, the vertically sown seeds had significantly enhanced germination rate compared to the horizontally sown seeds under the in vitro conditions, indicating that the seed sowing position affects the dandelion seed germination. These results are consistent with a previous study where sowing the seeds vertically gave higher germination rate than sowing the seeds flats in Jatropha plant $(\text { Derris indica })^{16}$. Particularly, horizontally downward orientation of the micropyle of the Jatropha plant seed significantly enhanced germination rate compared to upward or lateral orientations ${ }^{16}$. These results are expected since the seed micropyle plays essential roles in water uptake during germination ${ }^{17}$.

In this study, the germination rate of seed sown under the optimized in vitro conditions (vertically sowing seeds in media supplemented with $1.4 \mathrm{~g} / \mathrm{L}$ of MS and $1 \%$ of sucrose) was compared with the rate of seed sown in a soil pot (Figs. 5 and 6). The optimized in vitro germination conditions enhanced the germination rate $(64 \%)$ by almost 3 times compared to the rate (23\%) of seed sown on the soil pot (Figs. 5 and 6). Taken together, the current study demonstrates that the seed washing method and sowing position including concentration of MS and sucrose in media can be optimized to enhance in vitro seed germination of dandelion. It is concluded that the germination of

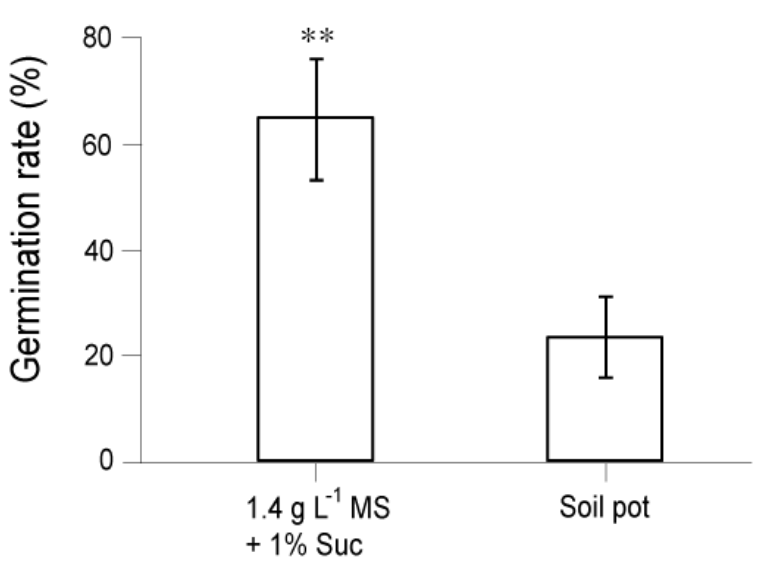

Fig. 6. Comparison of germination rates between seeds sown vertically on ' $A$ ' media supplemented with $1.4 \mathrm{~g} / \mathrm{L}$ of MS salts and $1 \%$ of sucrose (Suc), and in vivo seeds sown in a soil pot. The symbol $\left(^{* *}\right)$ indicate statistical significant difference at $p<0.01$.

dandelion seed can be maximized to as high as $64 \%$ by vertically sowing of seed under the in vitro culture conditions.

\section{ACKNOWLEDGEMENT}

This paper was supported by Wonkwang University in 2007.

\section{REFERENCES}

1. Schutz, K., Carle, R. and Schieber, A. (2006) Taraxacum-a review on its phytochemical and pharmacological profile, J. Ethnopharmacol. 107, 313-323.

2. Shin, S. R. (1999) Studies on the nutritional components of dandelion (Taraxacum officinale), Kor. J. Postharvest Sci. Technol. 6, 495-495.

3. Kim, K. H.,Chun, H. J. and Han, Y. S. (1998) Screening of antimicrobial activity of the dandelion (Taraxacum platycarpum) extract, Kor. J. Sco. Food Sci. Nutr. 14, 114-118.

4. Yun, S. I., Cho, H. R. and Cho, H. S. (2002) Anticoagulant from Taraxacum platycarpum, Biosci. Biotechnol. Biochem. 66, 1859-1864.

5. Ho, C., Choi, E. J., Yoo, G. S., Kim, K. M. and Ryu, S.Y. (1998) Desacetylmatricarin, an anti-allergic component from Taraxacum platycarpum, Planta Med. 64, 577-578.

6. Schippmann, U., Leaman, D. J. and Cunningham, A.B. (2002) Impact of Cultivation and Gathering 
of medicinal plants on Biodiversity: Global Trends and Issues. In: Biodiversity and the Ecosystem Approach in Agriculture, Forestry and Fisheries. FAO, 1-21.

7. Washitani, I. and Takenaka, A. (1987) Germination responses of Taraxacum platycarpum seeds to temperature, Plant Species Biol. 4, 123-130.

8. Canter, P. H, Thomas, H and Ernsta, E. (2005) Bringing medicinal plants into cultivation: opportunities and challenges for biotechnology, Trends Biotechnol. 23, 180-185.

9. Mogie, M. and Ford, H. (1988) Sexual and asexual Taraxacum species, Biol. J. Linn. Soc. 35, 155-168.

10. Marth, P. C. and Hamner, C. L. (1943) Vegetative propagation of Taraxacum kok-saghyz with the aid of growth substances, Bot. Gaz. 105, 35-48.

11. Liu, C. Z., Murch, S., Jain, J. C. and Saxena, P. K. (2004) Goldenseal (Hydrastis canadensis L.): In vitro regeneration for germplasm conservation and elimination of heavy metal contamination, In Vitro Cell. Dev. Biol. Plant 70, 73-79.

12. Lee, M. H., Yoon, E. S., Jung, S. J., Bae, K. H.,
Seo, J. W. and Choi, Y. E. (2002) Plant regeneration and effect of auxin and cytokinin on adventitious shoot formation from seedling explant of Taraxacum Platycarpum, Kor. J. Plant Biotechnol. 29, 111-115.

13. Todorović, S., Grubišić, D., Giba, Z., Mišić, D. and R. Konjević. (2006) Sucrose effects on in vitro fruiting and seed production of Centaurium pulchellum, Biol. Plant. 50, 771-774.

14. Lee, H. H., Kim, Y. S. and Park H. Y. (2007) Plant regeneratiopn via organogenesis from leaf explants culture of Taraxacum coreanum Nakai, Kor. J. Medicinal $15,62-66$.

15. Price, J., Li, T. C., Kang, S. G., Na, J. K. and Jang, J. C. (2003) Mechanisms of glucose signaling during germination of Arabidopsis. Plant Physiol. 132, 1424-1438.

16. Swaminathan, C., Vinaya Rai, R. S., Suresh, K. K. and Sivaganam, K. (1993) Improving seed germination of Derris indica by vertical sowing, J. Tropical Forest Sci. 6, 152-158.

17. Hamly, D. H. (1932) Softening of the seeds of Melilotus alba. Bot. Gaz. 93, 345-375. 